\title{
Initial clinical presentation of children with acute and chronic versus acute subdural hemorrhage resulting from abusive head trauma
}

\author{
Kenneth W. Feldman, MD, ${ }^{1}$ Naomi F. Sugar, MD, ${ }^{1}$ and Samuel R. Browd, MD, PhD² \\ 'Department of Pediatrics, Children's Protection Program, and 2Department of Neurological Surgery, Seattle Children's and \\ Harborview Medical Center, University of Washington, Seattle, Washington
}

\begin{abstract}
OBJECT At presentation, children who have experienced abusive head trauma (AHT) often have subdural hemorrhage $(\mathrm{SDH})$ that is acute, chronic, or both. Controversy exists whether the acute SDH associated with chronic SDH results from trauma or from spontaneous rebleeding. The authors compared the clinical presentations of children with AHT and acute SDH with those having acute and chronic SDH (acute/chronic SDH).
\end{abstract}

METHODS The study was a multicenter retrospective review of children who had experienced AHT during 2004-2009. The authors compared the clinical and radiological characteristics of children with acute SDH to those of children with acute/chronic SDH.

RESULTS The study included 383 children with AHT and either acute SDH $(n=291)$ or acute/chronic SDH $(n=92)$. The children with acute/chronic SDH were younger, had higher initial Glasgow Coma Scale scores, fewer deaths, fewer skull fractures, less parenchymal brain injury, and fewer acute noncranial fractures than did children with acute SDH. No between-group differences were found for the proportion with retinal hemorrhages, healing noncranial fractures, or acute abusive bruises. A similar proportion (approximately $80 \%$ ) of children with acute/chronic SDH and with acute SDH had retinal hemorrhages or acute or healing extracranial injures. Of children with acute/chronic SDH, $20 \%$ were neurologically asymptomatic at presentation; almost half of these children were seen for macrocephaly, and for all of them, the acute SDH was completely within the area of the chronic SDH.

CONCLUSIONS Overall, the presenting clinical and radiological characteristics of children with acute SDH and acute/ chronic SDH caused by AHT did not differ, suggesting that repeated abuse, rather than spontaneous rebleeding, is the etiology of most acute SDH in children with chronic SDH. However, more severe neurological symptoms were more common among children with acute SDH. Children with acute/chronic SDH and asymptomatic macrocephaly have unique risks and distinct radiological and clinical characteristics.

http://thejns.org/doi/abs/10.3171/2014.12.PEDS14607

KEY WORDS child abuse; abusive head trauma; subdural hemorrhage; chronic subdural hemorrhage; subdural rebleeding; acute and chronic subdural hemorrhage; trauma

$\mathrm{C}$ HILDREN evaluated for abusive head trauma (AHT) often have acute subdural hemorrhage (SDH), chronic SDH, or both acute and chronic SDH..$^{1,6,8-}$ ${ }^{10,13}$ In cases in which both acute and chronic SDH are present, the question often arises whether the acute SDH represents new abusive trauma or rebleeding into an area of chronic SDH, either spontaneously and/or from minor trauma. ${ }^{2,5,11,13}$ Literature documenting clinical presentations and associated clinical findings for children with acute/chronic SDH (acute/chronic SDH) is sparse. ${ }^{8,13} \mathrm{Re}-$ searchers Datta et al. found radiological findings suggestive of multiple events of bleeding at different times in $53 \%$ of children with SDH caused by AHT. ${ }^{8}$ The SDH was multifocal for $65 \%$. At presentation, these patients had ei-

ABBREVIATIONS AHT = abusive head trauma; $\mathrm{CPT}=$ child protection team; $\mathrm{GCS}$ = Glasgow Coma Scale; $\mathrm{SDH}$ = subdural hemorrhage .

SUBMITTED October 31, 2014. ACCEPTED December 31, 2014.

INCLUDE WHEN CITING Published online May 1, 2015; DOI: 10.3171/2014.12.PEDS14607.

DISCLOSURE The Matty Eappen Foundation provided partial funding but had no role in study design, execution, or development of this manuscript. Drs. Feldman and Sugar have provided child abuse consultation and expert witness. Dr. Browd has no financial relationships relevant to this article to disclose. 
ther symptoms of acute neurological injury or raised intracranial pressure. Bradford et al. noted that $16 \%$ of children with SDH caused by abuse experienced subdural rebleeding without evidence of new trauma but that all of them lacked neurological symptoms. ${ }^{6}$ Similarly, Ishak found that $62 \%$ of children with SDH caused by abuse who undergo reimaging within 1 year after injury sustain rebleeding without any evidence of new trauma (21\% of the total population of children with SDH caused by abuse) (G. Ishak, personal communication, 2014). All of these children also lacked neurological symptoms, and all but $7 \%$ experienced rebleeding only within the area of the prior $\mathrm{SDH}$.

On the basis of the above information, one would expect that if rebleeding into the area of a chronic SDH occurred either spontaneously or as a result of trivial/minor trauma, almost all acute SDHs should be entirely within the area of the chronic SDH. ${ }^{13}$ Other neuroimaging abnormalities, such as acute cerebral edema or intraparenchymal hemorrhage, should be lacking, and there should be no significant acute extracranial injuries. ${ }^{13}$ In addition, one would expect that most of these children would lack acute neurological symptoms and that any neurological symptoms observed would be the result of increased intracranial pressure resulting from mass effect from a large acute $\mathrm{SDH} .{ }^{13}$ Finally, one would expect that any acute SDH caused by rebleeding would occur within or immediately adjacent to the area of the preexisting chronic SDH, not in a separate, distinct location.

As part of a 5.5-year, multisite study evaluating whether the economic recession was associated with changing rates of AHT, demographic and clinical data were collected for 459 children with an unequivocal diagnosis of AHT. ${ }^{4}$ The objective of the study reported here was to compare clinical presentations and cranial and extracranial injuries in a subset of children with either acute/chronic SDH or acute $\mathrm{SDH}$. We hypothesized that if acute SDH in children with acute/chronic SDH resulted from new abusive trauma, their clinical presentations and rates of acute and healing extracranial injuries and retinal hemorrhage should not differ from those of children with only acute SDH. We also hypothesized that if the acute SDH occurred spontaneously or as a result of nonabusive trivial trauma, then these children should have few, if any, acute extracranial injuries and/or retinal hemorrhages and few, if any, healing injuries. Likewise, we hypothesized that they should lack acute neurological symptoms, beyond those attributable to increased intracranial pressure from the volume of the new bleeding. ${ }^{6,13}$ Finally, we hypothesized that if the acute SDH in children with acute/chronic SDH resulted from spontaneous hemorrhage or trivial trauma, it should occur within or immediately adjacent to the area of the chronic SDH (as opposed to an area distinctly separate from that of the chronic SDH).

\section{Methods}

This study is a subanalysis of a larger (parent) multisite study evaluating AHT rates over time. ${ }^{4}$ Data were collected through retrospective review of data from 4 participating hospitals (Seattle Children's and Harborview Medical Center, Seattle WA; Nationwide Children's Hospital,
Columbus, OH; Cincinnati Children's Hospital Medical Center; and Children's Hospital of Pittsburgh of UPMC and the Safer Center for Resuscitation Research). Each of the 4 study hospitals had a Child Protection Team (CPT), which is a multidisciplinary team that includes at least child abuse pediatricians and social workers. These teams provided consultation and case review to the child's treating team. A child was eligible for study inclusion if AHT was unequivocally diagnosed by the CPTs of the 4 hospitals from January 1, 2004, through June 31, 2009. Judgment that AHT was unequivocal was made by consensus of the CPTs of the contributing hospitals. The means by which this decision was made by the individual child's caretaking team and CPTs for the parent study was not recorded but typically depended on associated abusive injuries, evidence of significant traumatic head injury without a history or with a minor history of trauma, a behaviorally or developmentally implausible history, confessed or witnessed assaults, or some combination of these factors. Although not part of the diagnosis, a history of interpersonal violence was often present. The CPT's decision was also informed by police and protective services investigations. Use of a CPT consensus is a common way to define AHT for research purposes and one we have used previously. ${ }^{3,16}$ The clinical data reported reflect the information available at the time of the AHT diagnosis. The study did not include children who died before evaluation by a child abuse pediatrician. During the study period, all hospitals contributing data had an established CPT with stable personnel.

At all 4 study sites, institutional review board approval with informed consent waiver was obtained. Clinical data included the chief complaint/reason for presentation as provided in the caretaker's history and whether caretakers provided any history of trauma before cranial imaging. Extracranial injuries included nonskull fractures, abusive bruises, burns, oral injuries (such as intraoral lacerations or frenulum tears) or visceral injuries (as documented by imaging). Such injuries, if acute, were defined as "acute extracranial injuries." Acute fractures lacked radiological evidence of healing and/or were associated with symptoms or signs documenting their timing. "Healing extracranial injuries" included radiologically healing fractures or healing abusive skin injuries (lesion age was determined by history and/or appearance). "Parenchymal brain injury" was defined as cerebral edema, parenchymal contusion or hemorrhage, infarction, shear injury, or hypoxic-ischemic injury. Chronic SDHs were not considered evidence of prior abuse. As part of the parent study, chief complaints/ reasons for presentation were aggregated by a single researcher (Rachel Berger).

Classification of types and locations of intracranial hemorrhage was based on neuroimaging (CT and MRI) supplemented by clinical information. The initial CT scan, which was usually obtained shortly after the child's presentation to medical care, and initial MR images were used. Imaging studies were obtained at the treating clinicians' discretion. If any surgical intervention occurred, images preceding that surgery were evaluated. Data collected included presence or absence of cranial soft-tissue swelling, skull fracture, SDH/subarachnoid hemorrhage, cerebral edema, parenchymal contusion, or infarction. 
CT and MRI studies were interpreted by pediatric neuroradiologists during clinical care. According to interpretation of the attending pediatric neuroradiologists, all SDHs were defined by CT and MRI to be acute SDH only, chronic SDH only, acute/chronic SDH, or unknown. For the Seattle and Columbus sites, the radiologists' interpretations were supplemented by the clinical history and physical findings in assigning this SDH classification. The criteria by which each child's SDH was classified as acute or acute/chronic were not documented in the parent study. Likewise, data about density pattern of the SDH at presentation were not collected. Only children with acute SDH alone or acute/chronic SDH were included in the current analysis. Examples of children with evidence of acute and chronic SDH are provided in Figs. 1-3.

\section{Defining Location of Intracranial Hemorrhage}

For all study sites, the location of each acute SDH relative to the chronic SDH in children with acute/chronic SDH was defined by the radiology reports. As part of the parent study, the local investigators at the Columbus, Pittsburgh, and Seattle sites supplemented the radiology report by personal review of the images. Acute SDH was defined as "within" the area of the chronic SDH if it was entirely within or layering immediately above or below the chronic SDH. The acute SDH was defined as any SDH "separate" from the chronic SDH if any part of the acute SDH was in a distinctly separate or different location from the chronic SDH.

Chi-square descriptive statistics were used to assess differences between categorical variables, such as demographic, Glasgow Coma Scale (GCS) score, and clinical characteristics. Yates correction was used for small cells; $\mathrm{p}$ values and relative risks with $95 \%$ confidence intervals were calculated. Continuous age data were evaluated by t-test; $p$ values and relative risks with $95 \%$ confidence intervals were calculated. $\mathrm{p}$ values less than or equal to 0.05 were considered significant.

\section{Results \\ Patients}

The parent study cohort comprised 459 children with AHT: 409 (89.1\%) with acute SDH, chronic SDH, or acute/ chronic SDH. Of these, the subanalysis cohort comprised 383 children: 291 (63\%) with acute SDH and 92 (20\%) with acute/chronic SDH.

\section{Demographics and Morbidity and Mortality Rates}

The mean (SD) age of children with acute and chronic SDH was significantly younger than that of children with acute SDH: 6.21 (5.88) versus 11.04 (13.20) months, respectively, $\mathrm{p}<0.01$. These children were more likely to be male $(70.7 \%$ vs $54.9 \%, \mathrm{p}<0.01)$. No difference in the high rate of public insurance coverage between groups was found $(77.5 \%$ vs $82.5 \%, \mathrm{p}=0.40)$.

Severity and clinical consequences of injury were greater among children with acute $\mathrm{SDH}$, as evidenced by poorer GCS scores, than among those with acute/chronic SDH ( $<<0.01)$ (Table 1). Mortality rates were also higher among children with acute SDH than among those with acute/chronic SDH (21.3\% vs 5.4\%, respectively, $\mathrm{p}<0.01)$. The proportion of children who were admitted to an intensive care unit did not differ between those with acute SDH and those with acute/chronic SDH.

\section{Clinical Presentation}

Overall, the most common $(40.7 \%)$ reason for presentation for medical care was nonspecific neurological signs such as vomiting or seizures (Table 2). This presentation was more common among children with acute/chronic SDH than among those with acute SDH $(51.1 \%$ vs $37.5 \%$, respectively, $\mathrm{p}=0.03)$. Catastrophic symptoms such as cardiac or respiratory arrest or unresponsiveness led to the presentation of more children with acute SDH than
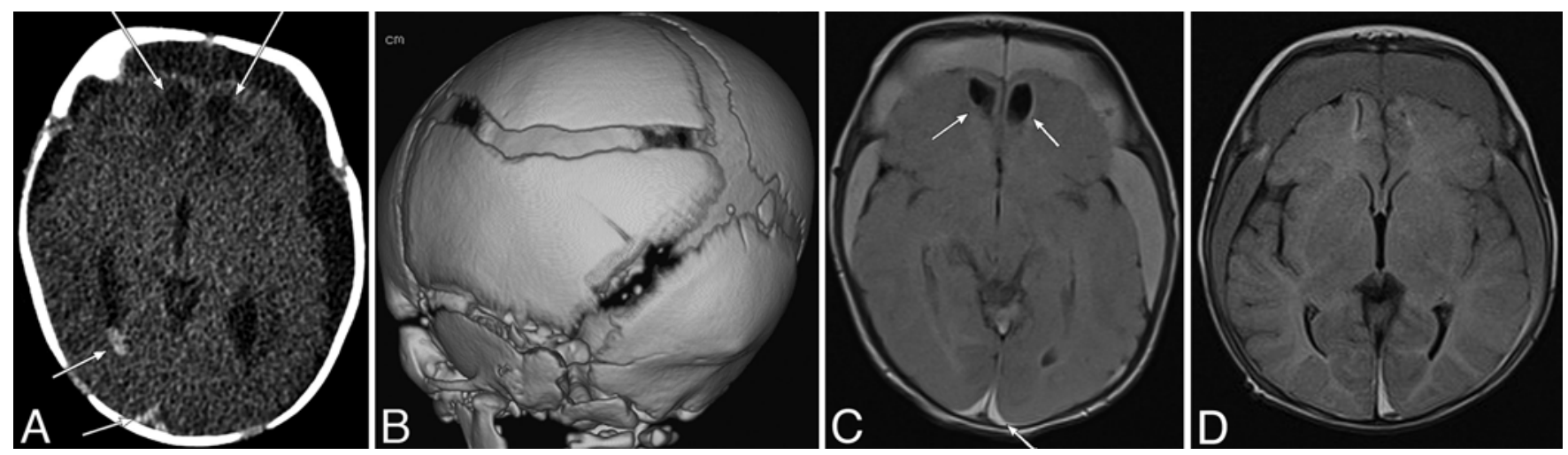

FIG. 1. Acute SDH separate from chronic SDH. The patient was born vaginally at 38 weeks of gestation without significant complications. At 1.5 weeks of age, while alone with her father, dark brown oral fluid began coming out of her mouth, after which ongoing emesis, lethargy, and irritability were observed. She presented at 3.5 weeks age with irritability, lethargy, intractable vomiting, and a bulging fontanelle. Head size was $95 \%$, and weight gain since birth was only 5 ounces. A few unilateral retinal hemorrhages but no fractures, bruises, or intraoral injury were found. A: Initial CT image showing bifrontal chronic SDH. Fresh hemorrhage (acute $\mathrm{SDH}$ ) can be seen in the posterior falx (short arrow), within the posterior horns of the lateral ventricles (short arrow), and around the cerebellum. Low-lying bifrontal cystic encephalomalacia was observed (long arrows). B: 3D skull shows diastatic sutures and a large horizontal diastatic right parietal fracture. C: MR image confirms that the SDH developed from multiple events at different times (posterior falx, more acute SDH, arrow) and shows contusional clefts (arrows). D: MR image showing new posterior fossa $\mathrm{SDH}$ (axial flair). 

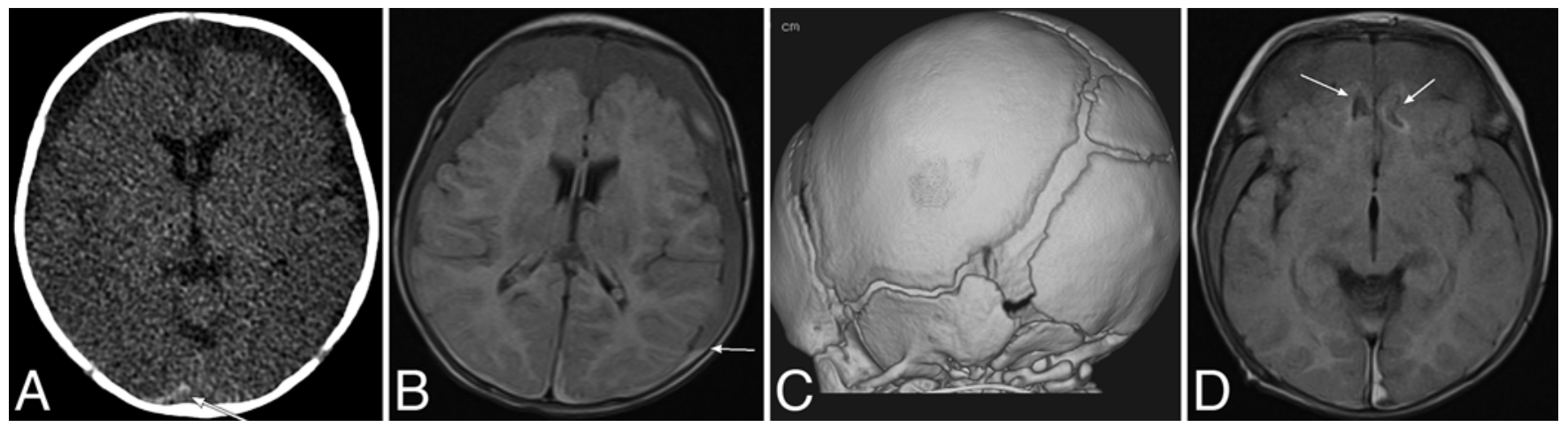

FIG. 2. Acute SDH within area of chronic SDH. The patient was a 2-month-old boy in whom apnea and limpness developed while he was with his father. He had been born by emergency caesarean section because of fetal distress. Occipital frontal circumference was stable at $50 \%$ since birth. He had been seen by his primary care physician and in an emergency department at 1.2 months of age after several days of projectile vomiting accompanied by new "cross eyes." Diagnosis at that time was reflux. At presentation for $\mathrm{AHT}$, he had bilateral healing lateral rib fractures and a healing left midclavicle fracture consistent with the timing of the chronic SDH. He had profuse bilateral retinal hemorrhages but no intraoral or skin injury. A: Initial CT image showing acute SDH in posterior falx (arrow) accompanying bifrontal chronic SDH. B: Axial T1-weighted MR image confirming more acute SDH in the posterior falx and within the left chronic SDH. Bifrontal chronic SDH is present, with a collection of more acute blood in the left anterior parietal region. Sediment consisting of red blood cells was seen posteriorly in the left chronic SDH (arrow). C: 3D skull showing split sutures and an occipital incal bone. D: Axial FLAIR MR image taken 1 week after hospital admission, showing bifrontal contusional clefts that developed (arrows).

with acute/chronic SDH (23.0\% vs $12.0 \%, \mathrm{p}=0.03)$. Of children with acute/chronic SDH, 17 (18\%) presented for a history of trauma alone, without specified neurological symptoms. Excluding these children who lacked symptom-based chief complaints, 58 (77.3\%) of 75 of children
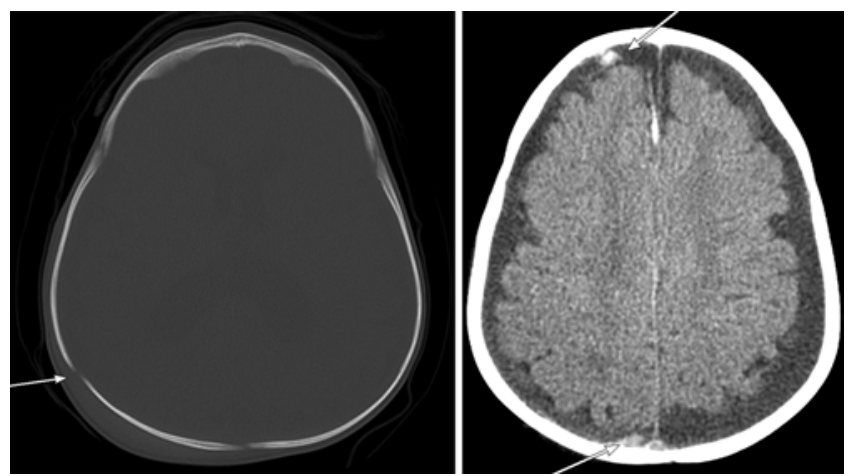

FIG. 3. Acute SDH within area of chronic SDH. The patient was a 10-month-old boy reported to have fallen from standing-adult height while under the care of a babysitter, who reported that he cried briefly, then became limp, pale, and was barely breathing. At the emergency room, his GCS score was 9, and brow bruising, swollen right parietaloccipital scalp, and extensive retinal hemorrhages were found. He had a subacute right zygomatic fracture. The emergency physician did not request head imaging. At 2 months of age, while with the same babysitter, he reportedly fell from a changing table. His pediatrician observed bruises and an ear infection. The next day he was lethargic; the emergency physician requested imaging of his head. From 2 to 10 months of age, his head size went from the 50th percentile to much greater than 95th percentile, for someone his age. Left: Bone window showing a large fracture (arrow) extending from his right occipital bone through his right parietal and frontal bones, accompanied by scalp swelling. Right: Bone window showing bilateral chronic SDHs containing small foci of acute hemorrhage over the right frontal cortex (arrow) and in the interhemispheric fissure and posterior falx (arrow). The babysitter subsequently admitted having pushed him violently to the floor, where he hit his head, after he grabbed her leg while wearing a soiled diaper. with acute/chronic SDH presented for acute neurological symptoms. This percentage did not differ from the 176 $(80.7 \%)$ of 218 children with acute SDH who presented for acute neurological symptoms $(\mathrm{p}=0.64$, relative risk $=$ 0.86 [95\% CI $0.54-1.36]$ ). Asymptomatic macrocephaly was the reason for presentation for more children with acute/chronic SDH than for those with acute SDH (7.5\% vs $1 \%, \mathrm{p}<0.01)$. Initial presentation without any history of trauma was more likely for children with acute/chronic SDH than for children with acute SDH $(79.4 \%$ vs $59.1 \%$, $\mathrm{p}<0.01)$.

\section{Neuroimaging}

All enrolled children underwent cranial CT, and 64\% underwent MRI. In every case, the MR images supported the finding of acute and chronic SDH shown on CT images. However, in the parent study, the relative contribution of CT and MR images to the child's evaluation was not documented. More radiological evidence of parenchymal brain injury was found for children with acute SDH than for children with acute/chronic SDH $(56.6 \%$ vs $39.1 \%$, respectively, p < 0.01) (Table 3). Children with acute SDH also had more skull fractures $(38.1 \%$ vs $21.7 \%$, p < 0.01$)$. Scalp soft-tissue swelling did not differ between the groups $(26.4 \%$ vs $26.3 \%$, $\mathrm{p}=0.90)$.

\section{Retinal Hemorrhage}

The frequency of retinal hemorrhage among children with acute SDH was similar to that among children with acute and chronic SDH (65.1\% vs $65.2 \%$, respectively, $\mathrm{p}=$ 0.89) (Table 3).

\section{Associated Extracranial Injuries}

Acute Associated Injuries

Acute extracranial fractures were more common among 
TABLE 1. Characteristics of children with acute or both acute and chronic SDH

\begin{tabular}{|c|c|c|c|c|}
\hline Characteristic & Acute SDH $(n=291)^{*}$ & Acute and Chronic SDH $(n=92)^{*}$ & $\mathrm{p}$ Value & Relative Risk $(95 \% \mathrm{Cl}) \ddagger$ \\
\hline GCS score & & & $<0.01$ & \\
\hline $3-7$ & $44.1 \%(104 / 236)$ & $24.0 \%(18 / 75)$ & & \\
\hline $8-12$ & $9.7 \%(23 / 236)$ & $13.3 \%(10 / 75)$ & & \\
\hline $13-15$ & $46.2 \%(109 / 236)$ & $62.7 \%(47 / 75)$ & & \\
\hline ICU admission & $70.6 \%(204 / 289)$ & $64.8 \%(59 / 91)$ & 0.36 & $1.09(0.92-1.29)$ \\
\hline Death & $21.3 \%(62)$ & $5.4 \%(5)$ & $<0.01$ & $3.92(1.63-9.46)$ \\
\hline
\end{tabular}

children with acute SDH than among children with acute and chronic SDH (30.7\% vs $18.8 \%$, respectively, $\mathrm{p}=0.05)$ (Table 3). The frequency of acute bruises/skin injury did not differ between children with acute SDH and those with acute and chronic SDH $(55.3 \%$ vs $50.1 \%, \mathrm{p}=0.51)$ nor did the frequency of any acute extracranial injuries $(72.0 \%$ vs $69.7 \%, \mathrm{p}=0.89$ ).

\section{Healing-Associated Extracranial Injuries}

No differences were found in the proportion of children with acute/chronic SDH and acute SDH who had healing fractures $(25.0 \%$ vs $33.1 \%$, respectively, $\mathrm{p}=0.17)$, healing skin injuries $(27.5 \%$ vs $17.6 \%, \mathrm{p}=0.07)$, or any healing extracranial injuries (36.9\% vs $37.1 \%, \mathrm{p}=0.92)$ (Table 3 ).

\section{Sum of Acute and/or Healing Extracranial Injuries or Retinal Hemorrhage}

Overall, acute or healing extracranial injuries and/or retinal hemorrhage were found in $78.6 \%$ of children. The proportion of children with acute SDH and acute/chronic $\mathrm{SDH}$ at the time of initial clinical presentation with these injuries did not differ $(81.8 \%$ vs $77.2 \%$, respectively, $\mathrm{p}=$ 0.409) (Table 3).

\section{Associations Between Acute and Chronic SDH Location and Chief Complaints/Reasons for Presentation}

Of 92 children with acute/chronic SDH, information about the site of acute SDH in association with the chronic SDH was available for 80 children; acute SDH was contained within the area of the chronic SDH in 43 cases (53.7\%). Any acute SDH separate from the chronic SDH was found in 37 cases (46\%). Table 4 compares presenting complaints/reasons for presentation, as related in the caretaker's initial history, for children with acute SDH within versus separate from the area of the chronic SDH. A history of acute neurological symptoms was found for $56 \%$ of children with an acute SDH within the area of the chronic SDH and $65 \%$ of children with an acute SDH separate from the area of the chronic SDH. Results of comparisons between groups were not significant, except for children with asymptomatic macrocephaly at presentation, for all of whom the acute SDH was entirely within the area of the chronic SDH.

\section{Discussion}

To the best of our knowledge, this subanalysis is the

TABLE 2. Chief complaints/reasons for presentation for children with either acute SDH or both acute and chronic SDH

\begin{tabular}{|c|c|c|c|}
\hline \multirow[b]{2}{*}{ Complaint/Reason } & \multicolumn{2}{|c|}{$\%$ (no.) of Patients } & \multirow[b]{2}{*}{$p$ Value* } \\
\hline & Acute SDH $(n=291)$ & Acute \& Chronic SDH $(n=92)$ & \\
\hline Cardiac or respiratory arrest or unresponsive & $23.0 \%(67)$ & $12.0 \%(11)$ & $<0.01$ \\
\hline Nonspecific neurological signs & $37.5 \%(109)$ & $51.1 \%(47)$ & 0.03 \\
\hline Altered mental state (not unresponsive) & $12.9 \%(35)$ & $13.0 \%(12)$ & \\
\hline Vomiting, ALTE, seizures, limpness, poor feeding & $20.3 \%(59)$ & $35.9 \%(33)$ & \\
\hline Respiratory distress & $5.2 \%(15)$ & $2.2 \%(2)$ & \\
\hline History of trauma, no specified signs or symptoms & $25.1 \%(73)$ & $18.5 \%(17)$ & 0.25 \\
\hline Miscellaneous complaints, neurologically asymptomatic & $10.0 \%(29)$ & $8.7 \%(8)$ & 0.88 \\
\hline Scalp soft-tissue swelling & $5.8 \%(17)$ & $1.1 \%(1)$ & \\
\hline Bruises & $4.1 \%(12)$ & $1.1 \%(1)$ & \\
\hline Sibling of abused child & $0 \%(0)$ & $1.1 \%(1)$ & \\
\hline Other & $3.4 \%(10)$ & $5.4 \%(5)$ & \\
\hline Macrocephaly & $1.0 \%(3)$ & $7.5 \%(7)$ & $<0.01$ \\
\hline
\end{tabular}


TABLE 3. Comparisons of associated injuries in children with acute SDH or both acute and chronic SDH*

\begin{tabular}{|c|c|c|c|c|}
\hline \multirow[b]{2}{*}{ Injury } & \multicolumn{2}{|c|}{$\%$ (no.) of Patients } & \multirow[b]{2}{*}{ p Value } & \multirow[b]{2}{*}{ Relative Risk $(95 \% \mathrm{Cl})$} \\
\hline & $\begin{array}{l}\text { Acute SDH } \\
(n=291)\end{array}$ & $\begin{array}{l}\text { Acute \& Chronic } \\
\text { SDH }(n=92)\end{array}$ & & \\
\hline \multicolumn{5}{|l|}{ Cranial injuries } \\
\hline Scalp soft-tissue swelling & $26.4 \%(67 / 254)$ & $26.3 \%(21 / 80)$ & 0.90 & $1.00(0.66-1.53)$ \\
\hline Skull fracture & $38.1 \%(111 / 291)$ & $21.7 \%(20 / 92)$ & $<0.01$ & $1.75(1.16-2.66)$ \\
\hline Parenchymal brain injury & $56.6 \%(164 / 290$ & $39.1 \%(36 / 92)$ & $<0.01$ & $1.18(1.05-1.33)$ \\
\hline Retinal hemorrhage & $65.1 \%(160 / 246)$ & $65.2 \%(43 / 66)$ & 0.89 & $1.00(0.82-1.22)$ \\
\hline \multicolumn{5}{|l|}{ Acute associated extracranial injuries $†$} \\
\hline Acute fractures (except skull) & $30.7 \%(78 / 254)$ & $18.8 \%(15 / 80)$ & 0.05 & $1.64(1.00-2.68)$ \\
\hline Acute bruises/skin injury & $55.3 \%(145 / 262)$ & $50.1 \%(38 / 75)$ & 0.51 & $1.05(0.93-1.18)$ \\
\hline Acute extracranial injury (fractures or bruises) & $69.8 \%(183 / 262)$ & $72.0 \%(54 / 75)$ & 0.83 & $0.98(0.86-1.10)$ \\
\hline \multicolumn{5}{|l|}{ Healing-associated extracranial injuriesł } \\
\hline Healing fractures (excluding skull) & $33.1 \%(84 / 254)$ & $25 \%(20 / 80)$ & 0.17 & $1.16(0.96-1.40)$ \\
\hline Healing skin injuries & $17.6 \%(44 / 250)$ & $27.5 \%(22 / 80)$ & 0.07 & $0.64(0.41-1.00)$ \\
\hline Healing fractures or skin injuries & $37.1 \%(108 / 291)$ & $36.9 \%(34 / 92)$ & 0.92 & $1.00(0.89-1.13)$ \\
\hline \multicolumn{5}{|l|}{$\begin{array}{l}\text { Sum of acute and/or healing extracranial injuries \&/ } \\
\text { or retinal hemorrhage }\end{array}$} \\
\hline Acute or healing skin injuries & $55.2(140 / 254)$ & $53.9(43 / 80)$ & 0.93 & $1.03(0.81-1.29)$ \\
\hline Acute or healing fractures & $40.5 \%(106 / 262)$ & $46.7 \%(35 / 75)$ & 0.41 & $0.94(0.84-1.06)$ \\
\hline $\begin{array}{l}\text { Any acute or healing extracranial injury or ret- } \\
\text { inal hemorrhage }\end{array}$ & $81.8 \%(238 / 291)$ & $77.2 \%(71 / 92)$ & 0.41 & $1.08(0.92-1.26)$ \\
\hline
\end{tabular}

largest study to compare presenting clinical and radiological findings of abused, head-injured children with documented acute SDH with findings of children with acute/ chronic SDH. Our data demonstrate that nearly $80 \%$ of children with acute/chronic SDH caused by AHT had evidence of either acute or healing extracranial injuries or retinal hemorrhage and that this proportion did not differ from the proportion of children with acute SDH alone. These data suggest that for most children, a new abusive event, rather than spontaneous rebleeding or minor trauma, is the cause of the acute SDH. If the acute SDH were caused by spontaneous rebleeding, one would not expect acute neurological symptoms, parenchymal brain injury, bruising, or fractures. ${ }^{13}$ The exception is the subgroup of children who were neurologically asymptomatic at presenting. This group included children evaluated for macrocephaly as well as a small number of children presented for miscellaneous complaints, such as scalp soft-tissue swelling, evaluation of the sibling of an abused child, strabismus, or isolated bruising. For these children, it is possible that the acute SDH may not have been the result of significant acute trauma. Bradford et al. reported

TABLE 4. Location of acute SDH relative to chronic SDH in relationship to complaints/reasons for presentation*

\begin{tabular}{lccccc}
\hline & \multicolumn{3}{c}{$\%$ (no.) of Patients } \\
\cline { 2 - 5 } \multicolumn{1}{c}{ Clinical Characteristic } & $\begin{array}{c}\text { Acute SDH Only } \\
\text { w/in Chronic } \\
\text { SDH }\end{array}$ & $\begin{array}{c}\text { Any Acute SDH } \\
\text { Separate From } \\
\text { Chronic SDH }\end{array}$ & $\begin{array}{c}\text { Total Acute \& } \\
\text { Chronic SDH }\end{array}$ & p Value & $\begin{array}{c}\text { Relative Risk } \\
(95 \% \text { Cl) }\end{array}$ \\
\hline Site of acute vs chronic SDH & $53.7 \%(43)$ & $46.3 \%(37)$ & $(80)$ & NA & NA \\
\hline Cardiac or respiratory arrest or unresponsive & $33.3 \%(2)$ & $66.7 \%(4)$ & $7.5 \%(6)$ & 0.54 & $0.60(0.19-1.90)$ \\
\hline Soft neurological signs & $53.5 \%(23)$ & $46.5 \%(20)$ & $53.8 \%(43)$ & 0.86 & $0.99(0.66-1.49)$ \\
\hline History of trauma, symptoms not described & $58.8 \%(10)$ & $41.2 \%(7)$ & $21.3 \%(17)$ & 0.84 & $1.12(0.71-1.78)$ \\
\hline Miscellaneous complaints & $14.3 \%(1)$ & $85.7 \%(6)$ & $8.8 \%(7)$ & 0.07 & $0.25(0.04-1.54)$ \\
\hline Macrocephaly & $100 \%(7)$ & $0 \%(0)$ & $8.7 \%(7)$ & 0.02 & $2.03(1.61-2.56)$ \\
\hline NA $=$ not applicable. & & & & &
\end{tabular}


the development of asymptomatic rebleeding in up to $16 \%$ of children with a prior acute SDH. ${ }^{6}$ Ishak has also observed that a minimum of $21 \%$ of children previously hospitalized for SDH from AHT subsequently rebleed. All of those rebleeding events were also asymptomatic (G. Ishak, personal communication, 2014). This finding is contrary to the finding that $77 \%$ of children with acute/chronic SDH in the current study and $100 \%$ of the children reported by Datta et al. ${ }^{8}$ had acute neurological symptoms at initial presentation. This difference fits our study postulate, in that Bradford et al. ${ }^{6}$ and Ishak were studying children with atraumatic rebleeding into existing SDHs, but we anticipated that we would be studying children with chronic SDH who had sustained new significant head and brain trauma causing additional acute SDH. Although bleeding in the head can be irritating, in these studies, it alone was not found to cause symptoms.

The presence of acute and chronic SDH at locations other than the site of the chronic SDH also supports our hypothesis that acute trauma rather than asymptomatic rebleeding accounts for the acute SDH in these children. When rebleeding occurs after minor or no trauma, it results from increased neovascular friability within the area of the chronic SDH neomembranes. ${ }^{13}$ Therefore, rebleeding should occur in locations in which chronic SDH is already present.

In our study, all of the children with acute/chronic SDH presenting for asymptomatic macrocephaly had acute SDH solely within the area of the chronic SDH. This finding suggests that if acute/chronic SDH is incidentally discovered in an otherwise asymptomatic child who undergoes a head CT solely because of an increased rate of head circumference growth, the acute SDH may have resulted from spontaneous chronic SDH rebleeding, particularly absent evidence of acute head trauma symptoms or other acute abusive injuries. The children in our study differ from those of McKeag et al. ${ }^{17}$ and Greiner et al. ${ }^{12}$ in that for the children in their studies, macrocephaly was caused by increased subarachnoid spaces, whereas for the children in our study, it was caused by chronic SDH. Development of acute SDH among 2\%-3\% of the children in these other studies suggests that increased extraaxial spaces may predispose to benign asymptomatic acute $\mathrm{SDH}$, albeit rarely. In children with either increased subarachnoid or subdural spaces, greater brain mobility could potentially cause new asymptomatic subdural bleeding at new sites.

Mortality rates were higher among children with acute SDH than among those with acute/chronic SDH. According to our findings, it is possible that children with acute SDH more frequently experienced a combination of cranial impact, as evidenced by more skull fractures, and acceleration-deceleration injury. This combination was associated with lower GCS scores, greater mortality rates, and more parenchymal brain injury among children with acute SDH. In contrast, at least a subset of the children with acute SDH may have followed the pattern reported by Adamsbaum et al., in which caretakers confessed repeated shaking events with or without impacts. ${ }^{1}$ Adamsbaum et al. reported that these events occurred over time and that the children did not present for medical attention initially but rather eventually, after the violent shaking caused symptoms requiring medical care. ${ }^{1}$ Although the greater frequency of skull fractures among older children could be explained if their skulls became more fragile with full ossification; contrary to that explanation, infant and toddler skull bone gains greater elastic modulus and ultimate strength with age ${ }^{7}$; among children younger than 18 months, two thirds of the skull fractures occurred before the children were 6 months of age..$^{14}$

Another cause of lower overall mortality rates among children with acute/chronic SDH is that this group included children with asymptomatic macrocephaly. None of these children died. However, exclusion of this subset of children does not account for all of the differences in mortality rates between children with acute SDH and those with acute/chronic SDH.

Although the most common cause of chronic SDH is trauma, both accidental and abusive, a variety of medical causes also exist. ${ }^{13}$ Most of these other causes are easily identified by associated history and clinical findings. ${ }^{13}$ The birth process is a well-recognized cause of acute SDH, but most hemorrhages occur at least partially in the posterior fossa and almost all resolve by 1 month of age and do not develop into chronic SDH. ${ }^{15,19,23}$ One study suggests that chronic SDH arises in newborns, but the means by which the etiologies of their chronic SDHs were diagnosed was not specified. ${ }^{18}$ No literature supports de novo development of postnatal chronic SDH in otherwise healthy infants. Because many of the findings of abuse are not immediately or clinically apparent (fractures and family violence) or are ephemeral (bruising and retinal hemorrhage), we feel that all infants presented for an unexplained chronic SDH should have a full abuse evaluation. The $37 \%$ rate of healing extracranial injuries among the children in our study with acute/chronic SDH suggests that their chronic SDHs were also traumatic.

\section{Study Limitations}

This study has several limitations. It was a secondary analysis of data collected for an observational epidemiological study. In defining acute $\mathrm{SDH}$ and acute/chronic $\mathrm{SDH}$, the child abuse pediatricians in the study used the attending neuroradiologists' clinical reports. Images were not evaluated by a single study radiologist or reviewed by more than 1 radiologist to determine interobserver reliability. However, the radiologists were all specialized, experienced radiologists who evaluate numerous cranial imaging studies as part of routine clinical practice. We recognize that it can be difficult to differentiate acute/chronic SDH from mixed-density acute SDH by SDH density alone on CT or MR images. ${ }^{6,20,21,22}$ However, older injuries are indicated by other imaging findings such as SDH membranes and septations, global or focal old parenchymal brain injuries, skull fractures without soft-tissue swelling, and signs of chronic intracranial pressure. At the Seattle and Columbus sites, for ultimate determinations of SDH age, the radiologists' determinations were supplemented by additional radiological findings and clinical information such as head-size growth patterns, history of past neurological events, character of drained SDH fluid, and pathology. It remains possible that some mixed-density acute SDHs were misclassified as acute/chronic SDHs. Any such misclassification would probably have biased our results toward the 
null hypothesis. If SDHs were misclassified, it is likely that other clinicians and radiologists would have similarly misclassified them, creating the same clinical issue of whether the intracranial injuries were both old and new.

We recognize that some nonskull fractures that we classified as acute because they lacked radiological changes of early healing could be up to 10 days old. ${ }^{21}$

Another limitation of our study relates to the inclusion criteria of the parent study, which required unequivocal AHT. Other children may have lacked sufficient signs or symptoms to undergo cranial imaging so that SDHs caused by AHT were not identified or AHT could not be unequivocally diagnosed. However, our approach should produce a valid series of children with a well-supported AHT diagnosis. AHT diagnosis often depends on the presence of other cranial and noncranial injuries. Dependence on these associated injuries for unequivocal diagnosis risks circularity in that it may result in higher rates of associated injuries for the children in our study than for children with AHT with more subtle injuries.

It is possible that a diagnosis of unequivocal abuse affected recognition of AHT differently for children with acute SDH than for those with acute/chronic SDH. Even among the children in our study, 20\% lacked additional abusive injuries but received a diagnosis of unequivocal AHT. Our retrospective data do not allow us to determine how the diagnoses were made.

\section{Conclusions}

In a large cohort of children with AHT, acute/chronic SDHs were present at initial clinical presentation for $20 \%$. Children with acute/chronic SDH at presentation in many ways did not differ from children with AHT and acute SDH only. The frequency of retinal hemorrhage, as well as the frequency of acute and healing extracranial injuries, did not differ between groups. The great frequency of healing extracranial injuries among children with acute/ chronic SDH suggests that the cause of the chronic SDH was prior trauma or abuse. The proportion of children with acute/chronic SDH and acute SDH presented with acute neurological symptoms did not differ, suggesting that the acute SDH in children with acute/chronic SDH is the result of new trauma rather than benign rebleeding.

However, the groups did differ in some respects. Initial GCS scores were lower; mortality rates were higher; and skull fractures, acute nonskull fractures, and parenchymal brain injury were more numerous among children with acute SDH than among children with acute/chronic SDH.

The group of children with asymptomatic macrocephaly, which accounted for nearly $9 \%$ of children with acute/ chronic SDH, was unique. In addition to these children being neurologically asymptomatic, the acute SDH for each child was entirely within the area of the chronic SDH. This finding suggests that in the scenario of asymptomatic macrocephaly and acute/chronic SDH, the acute SDH can occur spontaneously or after minor trauma.

\section{Acknowledgments}

Drs. Rachel Berger, Philip Scribano, and Kathi Makoroff served as local site principal investigators and were responsible for data abstraction and interpretation for the parent study. They provided editorial recommendations for this paper.

Dr. Sugar unfortunately died in July 2013, before completion of the final study editing.

\section{References}

1. Adamsbaum C, Grabar S, Mejean N, Rey-Salmon C: Abusive head trauma: judicial admissions highlight violent and repetitive shaking. Pediatrics 126:546-555, 2010

2. Barnes PD: Imaging of nonaccidental injury and the mimics: issues and controversies in the era of evidence-based medicine. Radiol Clin North Am 49:205-229, 2011

3. Berger RP, Dulani T, Adelson PD, Leventhal JM, Richichi R, Kochanek PM: Identification of inflicted traumatic brain injury in well-appearing infants using serum and cerebrospinal markers: a possible screening tool. Pediatrics 117:325-332, 2006

4. Berger RP, Fromkin JB, Stutz H, Makoroff K, Scribano PV, Feldman K, et al: Abusive head trauma during a time of increased unemployment: a multicenter analysis. Pediatrics 128:637-643, 2011

5. Block RW: Child abuse-controversies and imposters. Curr Probl Pediatr 29:249-272, 1999

6. Bradford R, Choudhary AK, Dias MS: Serial neuroimaging in infants with abusive head trauma: timing abusive injuries. J Neurosurg Pediatr 12:110-119, 2013

7. Coats B, Margulies SS: Material properties of human infant skull and suture at high rates. J Neurotrauma 23:12221232, 2006

8. Datta S, Stoodley N, Jayawant S, Renowden S, Kemp A: Neuroradiological aspects of subdural haemorrhages. Arch Dis Child 90:947-951, 2005

9. Ewing-Cobbs L, Prasad M, Kramer L, Louis PT, Baumgartner J, Fletcher JM, et al: Acute neuroradiologic findings in young children with inflicted or noninflicted traumatic brain injury. Childs Nerv Syst 16:25-34, 2000

10. Feldman KW, Bethel R, Shugerman RP, Grossman DC, Grady MS, Ellenbogen RG: The cause of infant and toddler subdural hemorrhage: a prospective study. Pediatrics 108:636-646, 2001

11. Fernando S, Obaldo RE, Walsh IR, Lowe LH: Neuroimaging of nonaccidental head trauma: pitfalls and controversies. Pediatr Radiol 38:827-838, 2008

12. Greiner MV, Richards TJ, Care MM, Leach JL: Prevalence of subdural collections in children with macrocrania. AJNR Am J Neuroradiol 34:2373-2378, 2013

13. Hymel KP, Jenny C, Block RW: Intracranial hemorrhage and rebleeding in suspected victims of abusive head trauma: addressing the forensic controversies. Child Maltreat 7:329348,2002

14. Laskey AL, Stump TE, Hicks RA, Smith JL: Yield of skeletal surveys in children $\leq 18$ months of age presenting with isolated skull fractures. J Pediatr 162:86-89, 2013

15. Looney CB, Smith JK, Merck LH, Wolfe HM, Chescheir NC, Hamer RM, et al: Intracranial hemorrhage in asymptomatic neonates: prevalence on MR images and relationship to obstetric and neonatal risk factors. Radiology 242:535-541, 2007

16. Maguire SA, Kemp AM, Lumb RC, Farewell DM: Estimating the probability of abusive head trauma: a pooled analysis. Pediatrics 128:e550-e564, 2011

17. McKeag H, Christian CW, Rubin D, Daymont C, Pollock AN, Wood J: Subdural hemorrhage in pediatric patients with enlargement of the subarachnoid spaces. J Neurosurg Pediatr 11:438-444, 2013

18. Parent AD: Pediatric chronic subdural hematoma: a retro- 
spective comparative analysis. Pediatr Neurosurg 18:266271,1992

19. Rooks VJ, Eaton JP, Ruess L, Petermann GW, Keck-Wherley J, Pedersen RC: Prevalence and evolution of intracranial hemorrhage in asymptomatic term infants. AJNR Am J Neuroradiol 29:1082-1089, 2008

20. Vinchon M, Noulé N, Tchofo PJ, Soto-Ares G, Fourier C, Dhellemmes P: Imaging of head injuries in infants: temporal correlates and forensic implications for the diagnosis of child abuse. J Neurosurg 101 (1 Suppl):44-52, 2004

21. Walters MM, Forbes PW, Buonomo C, Kleinman PK: Healing patterns of clavicular birth injuries as a guide to fracture dating in cases of possible infant abuse. Pediatr Radiol 44:1224-1229, 2014

22. Wells RG, Sty JR: Traumatic low attenuation subdural fluid collections in children younger than 3 years. Arch Pediatr Adolesc Med 157:1005-1010, 2003

23. Whitby EH, Griffiths PD, Rutter S, Smith MF, Sprigg A,
Ohadike P, et al: Frequency and natural history of subdural hemorrhages in babies and relation to obstetric factors. Lancet $363: 846-851,2003$

\section{Author Contributions}

Conception and design: Feldman, Sugar. Acquisition of data: Feldman, Sugar. Analysis and interpretation of data: all authors. Drafting the article: Feldman, Sugar. Critically revising the article: all authors. Reviewed submitted version of manuscript: Feldman, Browd. Approved the final version of the manuscript on behalf of all authors: Feldman. Statistical analysis: Sugar.

\section{Correspondence}

Kenneth Feldman, Children's Protection Program, Seattle Children's, 4800 Sand Point Way NE, Seattle, WA 98105. email: kfeldman@u.washington.edu. 